\title{
Journal of Bacteriology and

\section{Drug Resistance and Molecular Characteristics of Escherichia coli Isolates Associated with Acute Pyelonephritis}

\section{Xao-Li Cao ${ }^{\#}$, Xue-Jing Xü, Han Shen, Zhi-Feng Zhang, Ming-Zhe Ning, Jun-Hao Chen and Han Shen*}

Department of Laboratory Medicine, The Affiliated Nanjing Drum Tower Hospital of Nanjing University Medical School, Nanjing City, Jiangsu Province, People's Republic of China

\#Equal contributors.

"Corresponding author: Han Shen, Department of Laboratory Medicine, The Affiliated Nanjing Drum Tower Hospital of Nanjing University Medical School, Nanjing City, Jiangsu Province, People's Republic of China, Tel: + 8625831053 60, Fax: + 8625833071 15; E-mail: shenhan1036@sina.com

Received date: December 11, 2015; Accepted date: February 16, 2016; Published date: February 22, 2016

Copyright: (c) 2016 Cao XL, et al. This is an open-access article distributed under the terms of the Creative Commons Attribution License, which permits unrestricted use, distribution, and reproduction in any medium, provided the original author and source are credited.

\begin{abstract}
Acute pyelonephritis (APN) as one of the most severe form of UTIs may result in significant morbidity. We aim to investigate the antimicrobial susceptibilities and genetic traits of Escherichia coli isolates associated with APN.

Totally, 64 APN E. coli isolates were analyzed for the antimicrobial susceptibilities, phylogenetic groups, resistance and virulence determinants, plasmid replicons, pulsed-field gel electrophoresis (PFGE), and Multi-locus sequence types (MLST).

High percentages of resistance $(>65.0 \%)$ to ampicillin/sulbactam and levofloxacin were observed, imipenem and fosfomycin displayed good in vitro sensitivity (>93.0\%). Most of the strains belonged to phylogenetic group $D$ $(50.6 \%)$ and B2 $(21.6 \%)$, D strains were more resistant than B2 ones towards the cephalosporins tested $(p<0.05)$. Thirty-six (56.3\%) blaCTX-M, $3(4.7 \%) \mathrm{rmtB}$, and 13 plasmid mediated quinolone resistance (PMQR) genes were identified. Plasmid replicon IncF $(54 / 64,84.4 \%)$ and virulence factors (VFs) fimH $(57 / 64,89.1 \%)$ was the most prevalent. PFGE and MLST displayed genetic diversity. Prevalence of ompT, fdeC, PAI, and usp were higher among B2 strains than that in $D$ ones $(P<0.05)$. Statistical associations between antimicrobial resistances and VFs were found.

This study provides new data on the molecular epidemiology and pathogenesis of $E$. coli isolates associated with APN.
\end{abstract}

Keywords: Acute pyelonephritis; Antimicrobial resistance; Phylogenetic groups; Virulence genes; Resistance determinants; Escherichia coli

\section{Introduction}

Acute pyelonephritis (APN) as an acute infection of the upper urinary tract is frequently caused by Escherichia coli (56-85\%) when the bacteria in the bladder ascend the ureters and invade the kidneys, [1] with the possibility of causing significant morbidity and irreversible kidney damage. The genetic flexibility and the ability of $E$. coli to adapt to constantly changing environments allow the bacterium to acquire numerous antimicrobial resistance determinants such as extendedspectrum $\beta$-lactamases (ESBLs) and 16S rRNA methyltransferases (16S-RMTases) [2] and plasmid-mediated quinolone resistance (PMQR) genes are also frequently detected [3]. Thus, the production of these resistance determinants is a significant threat to patients with APN before the results of antibiotic susceptibility tests become available [1]. Moreover, mobile genetic elements such as plasmids have been reported to be associated with the dissemination of these resistance determinants among clinical strains [4]. However, limited data are available on the prevalence of ESBLs, PMQRs and 16SRMTase and the distribution of plasmid replicons among E. coli isolates associated APN.
The pathogenicity of $E$. coli strains has also been reported to be strongly influenced by virulence factors (VFs) including adhesion molecules (e.g., P fimbriae), toxins (e.g., hemolysin and cytotoxic necrotizing factor), iron-acquisition systems (e.g., the aerobactin system), protectins (e.g., $k p s M$ and traT), miscellaneous virulence determinants (e.g., usp and $m a l X$ ), cytolethal distending toxin, uropathogenesis-specific proteins, and the formation of biofilms [5,6] Furthermore, studies on E. coli strains isolated from Europe and USA have revealed that more VFs are distributed among phylogenetic group $\mathrm{B} 2$ and $\mathrm{D}$ strains than those among $\mathrm{A}$ and B1 strains $[7,8]$. However, such studies were rarely performed on $E$. coli strains associated with APN in China since the VFs and phylogenetic groups of such strains remain unclear.

In this study, we analyzed the molecular epidemiology and pathogenesis of $E$. coli isolates associated with APN. The relationship between distinct phylogenetic groups and resistance to antimicrobial agents as well as VFs in E. coli strains was analyzed. In addition, the association between antimicrobial resistance and the presence of VFs was also investigated. 


\section{Materials and Methods}

\section{Bacterial isolates}

Totally, 64 E. coli isolates were collected from 64 patients diagnosed as APN which was defined as the presence of two of the following syndromes: (a) axillary temperature $\geq 38.3^{\circ} \mathrm{C}$ or chills; (b) flank pain or costovertebral angle tenderness or pain on bimanual palpation of the kidney; and (c) mictional syndrome (including two or more of the following: dysuria, frequency, suprapubic pain or urgency), together with the presence of pyuria (a positive leukocyte esterase dipstick test result, subsequently confirmed by urinalysis with more than 10 leukocytes $/ \mathrm{mL}$ in urine without centrifuging or more than 5 leukocytes per high-power field in centrifuged sediment) or a positive urine culture. The patients were admitted during 2012-2013, and Standard biochemical tests were used for the isolation and identification of $E$. coli strains obtained in clinical microbiology laboratories of Nanjing Drum Tower Hospital affiliated to Nanjing University, Jiangsu, China.

\section{Antimicrobial susceptibility testing}

The susceptibility of the 64 strains toward 16 antimicrobial agents were tested by the Kirby-Bauer's disk-diffusion method and the results were interpreted according to Clinical and Laboratory Standards Institute (CLSI) guidelines [9]. The following antimicrobial agents ( $\mu \mathrm{g} /$ disk) were used: ampicillin/sulbactam (SAM, 10/10 $\mu \mathrm{g}$ ), cefuroxime (CXM, $30 \mu \mathrm{g}$ ), ceftazidime (CAZ, $30 \mu \mathrm{g}$ ), cefotaxime (CTX, $30 \mu \mathrm{g})$, cefepime (FEP, $30 \mu \mathrm{g}$ ), cefoxitin (FOX, $30 \mu \mathrm{g}$ ), aztreonam (ATM, 30 $\mu \mathrm{g})$, imipenem (IPM, $10 \mu \mathrm{g}$ ), amikacin (AK, $30 \mu \mathrm{g})$, trimethoprimsulfamethoxazole (SXT, 1.25/23.5 $\mu \mathrm{g}$ ) levofloxacin (LEV, $5 \mu \mathrm{g}$ ), piperacillin (PRL, $100 \mu \mathrm{g}$ ), cefoperazone/sulbactam (SCF, 75/30 $\mu \mathrm{g}$ ), ticarcillin/clavulanic acid (TLM, 75/10 $\mu \mathrm{g}$ ), piperacillin/tazobactam (TZP, 100/30 $\mu \mathrm{g}$ ) and fosfomycin (FOM, $200 \mu \mathrm{g}$ ). The disks were obtained from Oxoid Ltd. (Basingstoke, Hampshire, England). E. coli ATCC 25922 was used as the quality control in parallel.

\section{Assignment of phylogenetic groups}

The 64 strains were assigned to phylogenetic groups $\mathrm{A}, \mathrm{B} 1, \mathrm{~B} 2$, or D by using a triplex PCR-based strategy with specific primers for $c h u A$, yja $A$, and $T s p E 4$. C2 determinants according to a previously described protocol [10].

\section{Identification of resistance genes}

The strains displaying non-susceptibility to cefotaxime or cefepime were screened for ESBLs encoding genes including blaCTX-M, blaTEM, and blaSHV genes according to the PCR protocol described previously [11]. The strains that survived on Luria-Bertani plates containing $0.125 \mu \mathrm{g} / \mathrm{mL}$ ciprofloxacin were screened for PMQR genes as previously described [12-14]. Strains resistant to amikacin were analyzed for the presence of 16S-RMTases [15]. PCR and sequencing (both strands) were used to analyze the genes, the nucleotide and deduced amino acid sequences were compared with those available in the GenBank database.

\section{Plasmid incompatibility group}

Plasmid replicon typing was performed in order to establish the range and diversity of plasmids amongst $E$. coli isolates associated with APN. Highly pure total DNA was prepared using the Wizard ${ }^{\oplus}$ Genomic DNA purification Kit (Promega) according to the manufacturer's procedure. Plasmids were typed using the PCR-based replicon typing method; 5 multiplex and 3 simple PCR amplification steps were used with specific primers and conditions, as described by Carattoli et al. [16].

\section{Virulence genotyping}

Multiplex PCR assays were performed with specific primers to check for the presence of 15 genes that have been reported to be associated with virulence in $E$. coli isolates: iut $A$, ompT, fyuA, fdeC, fimH, traT, cvaC, pap, kpsMT, pAI, usp, aer, hlyA, cnf, and chuA [17]. chuA was detected by triplex PCR as mentioned above.

\section{Multi-locus sequence typing}

The sequence types (STs) of 64 strains were analyzed by amplifying and sequencing 7 housekeeping genes, including $a d k$, fum $C$, gyr $B$, icd, $m d h$, purA, and $\operatorname{rec} A$ according to the protocol available at http:// mlst.ucc.ie/mlst/dbs/Ecoli.

\section{Strain typing by pulsed-field gel electrophoresis (PFGE)}

All the 64 strains associated with APN were analyzed for genetic relatedness by using $\mathrm{XbaI}$ according to the U.S. CDC PulseNet protocol [18]. Briefly, electrophoresis was performed with the switch time from 2.2 to $54.2 \mathrm{~s}$ at a gradient of $6 \mathrm{~V} / \mathrm{cm}$ and an included angle of $120^{\circ}$ for $19 \mathrm{~h}$ after the bacterial chromosome was digested by $\mathrm{XbaI}$. The analysis of the PFGE profiles was performed by Bionumerics software v6.5 (Applied Maths, Sint-Martens-Latem, Belgium) using the Dice similarity coefficient on the basis of the unweighted-pair group method using average linkages (UPGMA), with a $1.5 \%$ band tolerance. And genetic relatedness was observed with the cutoff lines at $85 \%$ and $69 \%$.

\section{Statistical analysis}

The statistical analysis was performed by using SPSS software version 20 (SPSS Inc., Chicago, IL, USA). Proportions were compared using the chi-squared test or, when the expected numbers were $<5$, Fisher's exact test (two-tailed). $\mathrm{P}<0.05$ was considered statically significant.

\section{Results}

\section{Antimicrobial resistance of $E$. coli isolates associated with acute pyelonephritis}

The E. coli isolates exhibited the highest resistance rate toward SAM (59/64, 92.6\%) followed by LEV (42/64, 65.6\%), CXM (34/64, 53.1\%), SXT $(35 / 64,54.7 \%)$, PRL $(33 / 64,51.6 \%)$, CTX (31/64, 48.4\%), TLM $(31 / 64,48.4 \%)$, and FEP $(21 / 64,32.8 \%)$ TZP $(28 / 64,43.8 \%)$. By comparison, the resistance rates were relatively lower toward $\mathrm{AK}$ (19/64, 29.7\%), CAZ (14/64, 21.9\%), FOX (8/64, 12.5\%), and SCF $(7 / 64,10.9 \%)$. Good in vitro sensitivity towards IPM $(2 / 64,3.1 \%)$ and FOM $(4 / 64,6.3 \%)$ was observed.

\section{Assignment of phylogenetic groups}

Among the $64 \mathrm{E}$. coli isolates tested, 30 (50.6\%) strains were assigned to phylogenetic group D, 19 (21.6\%) to B2, 8 (12.5\%) to A, and $7(8.5 \%)$ to B1. As shown in Table 1, the D strains exhibited higher resistances than the B2 strains did toward CTX (50.0\% vs. $10.5 \%$, 
Citation: Cao XL, Xu XJ, Shen H, Zhang ZF, Ning MZ, et al. (2016) Drug Resistance and Molecular Characteristics of Escherichia coli Isolates

Page 3 of 6

$\mathrm{P}=0.012$ ), $\mathrm{PRL}$ (63.3\% vs $21.1 \%, \mathrm{P}=0.009$ ), $\mathrm{FEP}$ (60.0\% vs $26.3 \%$, $\mathrm{P}=0.021)$, and $\operatorname{ATM}(63.3 \%$ vs $15.8 \%, \mathrm{P}=0.003)$.

\section{Prevalence of antimicrobial resistance determinants}

On the whole, 36 (56.3\%) blaCTX-M including 18 blaCTX-M-14, 13 blaCTX-M-15, 3 blaCTX-M-27, and 1 blaCTX-M-55 as well as 1 blaCTX-M-3 were identified; 37(57.8\%) blaTEM-1 including 36 blaTEM-1 and 1 blaTEM-104 as well as $1(1.6 \%)$ blaSHV-1 were also detected.
Among the 16S-RMTase-encoding genes, 3 (4.7\%) rmtB genes were identified in the 19 isolates resistant to amikacin.

The PMQR genes were identified in the following isolates: 6 (9.4\%) aac( $\left.6^{\prime}\right)-I b-c r, 3$ (4.7\%) qnr (1 qnrB6 and $1 q n r S 11$ as well as $\left.1 q n r S 2\right)$ and 4 efflux pumps genes including 3 (4.7\%) oqx $A B$ and 1 (1.7\%) qepA.

\begin{tabular}{|c|c|c|c|c|c|c|}
\hline \multirow{2}{*}{$\begin{array}{l}\text { Antimicrobial } \\
\text { agents }\end{array}$} & \multicolumn{4}{|c|}{ Prevalence of resistant strains, no. (Column \%) } & \multicolumn{2}{|l|}{$P$ value } \\
\hline & $A(N=8)$ & $B 1(N=7)$ & $B 2(N=19)$ & $\mathrm{D}(\mathrm{N}=30)$ & D vs B2 & $D$ vs $A \& B 1$ \\
\hline SAM & $8(100)$ & $7(100.0)$ & $17(89.5)$ & $27(90.0)$ & 1.000 & 0.540 \\
\hline AK & $2(25.0)$ & $2(28.6)$ & $5(26.3)$ & 10(33.3) & 0.604 & 0.909 \\
\hline CAZ & $3(37.5)$ & $1(14.3)$ & $1(5.3)$ & $9(30.0)$ & 0.084 & 1.000 \\
\hline CXM & $6(75.0)$ & $4(57.1)$ & $11(57.9)$ & $23(76.7)$ & 0.165 & 0.475 \\
\hline FEP & $2(25.0)$ & $2(28.6)$ & $2(10.5)$ & $15(50.0)$ & 0.012 & 0.240 \\
\hline FOX & $1(12.5)$ & $1(14.3)$ & $0(0.0)$ & $6(20.0)$ & 0.069 & 0.890 \\
\hline IPM & $1(12.5)$ & $0(0.0)$ & $0(0.0)$ & $1(3.3)$ & 1.000 & 1.000 \\
\hline SCF & $2(25.0)$ & $0(0.0)$ & $1(5.3)$ & $4(13.3)$ & 0.671 & 1.000 \\
\hline TZP & $2(25.0)$ & $2(28.6)$ & $10(32.6)$ & $10(33.3)$ & 0.181 & 0.909 \\
\hline PRL & $7(87.5)$ & $3(42.9)$ & $4(21.1)$ & $19(63.3)$ & 0.009 & 0.826 \\
\hline CTX & $6(75.4)$ & $2(28.6)$ & $5(26.3)$ & $18(60.0)$ & 0.021 & 0.670 \\
\hline TIM & $2(25.0)$ & $2(28.6)$ & $12(63.2)$ & $15(50.0)$ & 0.367 & 0.240 \\
\hline LEV & $5(62.5)$ & $6(85.7)$ & $12(63.2)$ & $19(63.3)$ & 0.990 & 0.737 \\
\hline SXT & $4(50.0)$ & $4(57.1)$ & $10(52.6)$ & $17(56.7)$ & 0.782 & 0.832 \\
\hline ATM & $4(50.0)$ & $4(57.1)$ & $3(15.8)$ & $19(63.3)$ & 0.003 & 0.519 \\
\hline FOM & $1(12.5)$ & $0(0.0)$ & $1(5.3)$ & $2(6.67)$ & 1.000 & 1.000 \\
\hline
\end{tabular}

Table 1: Association between resistance to antimicrobial agents and phylogenetic groups of Escherichia coli isolates associated with acute pyelonephritis.

\section{Plasmid replicons}

On the whole, 60/64 (93.8\%) strains contained plasmid replicons and IncF was the most prevalent replicon $(54 / 64,84.4 \%)$, followed by IncI1 (32/64, 50.0\%); 3 IncP (4.7\%), 1 IncN (1.6\%), and 1 IncA/C (1.6\%) replicons were also detected. Notably, 30 strain (46.9\%) carried 2 plasmid replicons.

The distributions of plasmid replicons among the group B2 E. coli isolates $(18 / 19,94.7 \%)$ was higher than that among group D isolates $(19 / 30,63.3 \%)(P=0.032)$. Resistance to SAM $(P=0.027)$ and AK $(P=0.026)$ were obviously higher among IncF positive strains than IncF negative ones. Significant association between the distribution of IncI1 and resistance to CAZ $(\mathrm{P}=0.034), \operatorname{CXM}(P=0.035)$, and CTX $(P=0.024)$ were found.
In addition, the distribution of traT was higher among the IncF replicon-positive strains than that among IncF replicon-negative strains: $(87.0 \%$ vs $0.0 \%, P=0.000)$. Whereas, the distribution of PAIs was higher among the IncF replicon-negative strains than among IncF replicon-positive strains: $(40.0 \%$ vs $7.4 \%, P=0.019)$. The distribution of all the virulence genes were independent of IncI1 replicons.

\section{Distribution of virulence genes}

Multiple VFs were identified among the strains examined in our study. All the strains carried at least 1 adhesion-associated gene with the fimH $(57 / 64,89.1 \%)$ and $f d e C(45 / 64,70.3 \%)$ being the most prevalent genes, followed by pap (14/64, 21.9\%). traT, which encodes a protectin, was detected in $47(73.4 \%)$ strains, and the iron uptake- 
Page 4 of 6

associated genes fyuA $(42 / 64,65.6 \%)$, iutA $(41 / 64,64.1 \%)$, and $k p s M T$ (34/64, 53.1\%) were also identified. chuA was also found among 49 (76.6\%) out of 64 strains. By comparison, other virulence genes such as ompT $(28 / 64,44.3 \%)$, usp $(15 / 64,20.5 \%)$, PAIs $(8 / 64,15.9 \%)$, and cvaC $(2 / 64,3.4 \%)$ were less common, and aer, hlyA, and cnf were not detected among our strains. We also found that 57 (89.1\%) strains carried $\geq 3$ VFs.
As shown in Table 2, ompT, fdeC, PAI, and usp were more prevalent among $\mathrm{B} 2$ strains than among $\mathrm{D}$ strains $(\mathrm{P}<0.05)$, whereas the distribution of iutA, ompT, fyuA, fdeC, traT, and kpsMT was higher in group $\mathrm{D}$ than that in groups $\mathrm{A}$ and $\mathrm{B} 1(\mathrm{P}<0.05)$.

\begin{tabular}{|l|l|l|l|l|l|l|}
\hline \multirow{2}{*}{ Virulence genes } & \multicolumn{2}{|l}{ Prevalence of gene, no. (Column \%) } & \multicolumn{2}{l|}{ P value } \\
\cline { 2 - 7 } & $\mathrm{A}(\mathrm{N}=8)$ & $\mathrm{B} 1(\mathrm{~N}=7)$ & $\mathrm{B} 2(\mathrm{~N}=19)$ & $\mathrm{D}(\mathrm{N}=30)$ & D vs B2 & D vs A\& B1 \\
\hline iutA & $3(37.5)$ & $2(28.6)$ & $15(78.9)$ & $21(70.0)$ & 0.719 & 0.043 \\
\hline ompT & $0(0.0)$ & $2(28.6)$ & $14(73.7)$ & $12(40.0)$ & 0.021 & 0.139 \\
\hline fyuA & $1(12.5)$ & $1(14.3)$ & $16(84.2)$ & $24(80.0)$ & 1.000 & 0.000 \\
\hline fdeC & $6(75.0)$ & $6(85.7)$ & $17(89.5)$ & $16(53.3)$ & 0.021 & 0.158 \\
\hline fimH & $6(75.0)$ & $6(85.7)$ & $18(94.7)$ & $27(90.0)$ & 0.956 & 0.642 \\
\hline traT & $4(50.0)$ & $4(57.1)$ & $12(63.2)$ & $27(90.0)$ & 0.056 & 0.016 \\
\hline cvaC & $0(0.0)$ & $0(0.0)$ & $1(5.3)$ & $1(3.3)$ & 1.000 & 1.000 \\
\hline kpsMT & $0(0.0)$ & $1(14.3)$ & $12(63.2)$ & $21(70.0)$ & 0.619 & 0.000 \\
\hline pap & $0(0.0)$ & $1(14.3)$ & $7(36.8)$ & $6(20.0)$ & 0.913 & 0.647 \\
\hline PAI & $0(0.0)$ & $0(0.0)$ & $7(36.8)$ & $1(3.3)$ & 0.007 & 1.000 \\
\hline usp & $0(0.0)$ & $0(0.0)$ & $15(78.9)$ & $0(0.0)$ & 0.000 & 1.000 \\
\hline chuA & $0(0.0)$ & $0(0.0)$ & $19(100.0)$ & $30(100.0)$ & ND & ND \\
\hline ND: not determined. & & & & & \\
\hline
\end{tabular}

Table 2: Phylogenetic group distribution of virulence-associated genes among 64 Escherichia coli isolates associated with acute pyelonephritis.

Additionally, the distribution of ompT and $k p s M T$ was higher among levofloxacin resistant strains than that among levofloxacin susceptible strains: $(72.7 \%$ vs $38.1 \%, 72.7 \%$ vs $42.9 \%$, P0.05). There were statistically significant associations between $\operatorname{tra} T$ gene and resistances to $\mathrm{PRL},(\mathrm{P}=0.033)$, and $\mathrm{ATM},(\mathrm{P}=0.0011)$. Such associations were also found between usp gene and resistances to $\mathrm{CXM},(\mathrm{P}=0.023)$, TZP ( $\mathrm{P}=0.041)$, PRL, $(\mathrm{P}=0.002)$, and ATM $(\mathrm{P}=0.007)$ as well as between chuA gene and CTX $(\mathrm{P}=0.035)$. Significant associations between iutA gene and resistances to TZP, $(\mathrm{P}=0.001)$, and TLM, $(\mathrm{P}=0.048)$ was observed, in addition to the associations between $\operatorname{omp} T$ gene and PRL $(\mathrm{P}=0.025)$ and $\mathrm{AK}(\mathrm{P}=0.008)$ as well as between fdeC gene and $\mathrm{CXM}(\mathrm{P}=0.046)$.

\section{MLST}

MLST analysis identified 16 different STs with ST131 (10, 15.6\%), ST38 (8, 12.5\%), ST69 (8, 12.5\%), ST648 (8, 12.5\%), ST405 (6, 9.38\%)ST10 $(\mathrm{n}=5)$ being the major STs which accounted for $70.3 \%$ $(45 / 64)$ of the isolates. In addition, ST73 $(n=3)$, ST14 $(n=2)$, ST155 $(n=2)$, ST393 $(n=2)$, ST394 $(n=2)$, ST410 $(n=2)$, ST12, ST31, ST95, ST101, ST393, ST522 were also found, indicating a diverse lineages.

\section{PFGE}

PFGE displayed that 64 E. coli isolates associated with APN showed a genetic diversity, indicating that they were not clonally related.

\section{Discussion}

The goal of this study was to assess the antimicrobial susceptibility $E$. coli associated with APN, and to explore the correlations between antimicrobial resistance and genetic traits including plasmid replicons, virulence factor possession, as well as $E$. coli phylogenetic group.

The results showed that, in contrast to the low resistance rates displayed by UPEC isolated from patients in USA and Europe, [19,20] the strains examined in our study exhibited a high frequency of resistance toward multiple antimicrobial agents, which agrees with previous reports from China [21] and is similar to that has been reported in India [22]. This seriously limits the choice of treatments for UTIs and poses a serious challenge to public health. Such a high level of resistance might result from the high proportion of blaESBLs identified in our collection, indicating that APN caused by ESBLproducing E. coli are a great concern. Fortunately, imipenem and fosfomycin still can be available because of the good in vitro sensitivity. Furthermore, the prevalence of $r m t B$ and PMQR genes in our isolates was lower than that in MDR E. coli isolates [23]. However, the particular concern is that the presence of these determinants might facilitate the development of multi-drug resistant strains under the selection pressure imposed by the antimicrobial agents used frequently in hospitals.

As reported previously [2], strains of phylogenetic group D were the most prevalent strains in our study. Notably, the group D strains in our 
study were more resistant toward cephalosporin than were group B2 strains. To the best of our knowledge, this observation has not been reported previously. Nevertheless, the B2 strains exhibited a higher virulence potential than the $\mathrm{D}$ strains did, albeit both of these phylogenetic groups were more virulent than A and B1 strains. This is in accordance with the results of multiple studies conducted in Europe [7-8].

The replicon type that was most highly represented among our strains was IncF, which is consistent with previously reported results [24]. This finding suggests that plasmid replicon IncF may be welladapted to E. coli strains. Furthermore, the IncI1 replicons identified here were also frequently detected in our previous study [25], which might result from the negligible fitness costs imposed by the IncI1 plasmid replicon on its E. coli host, as demonstrated in vitro by Fischer et al. [26]. The other replicons detected in this study have also been reported previously in E. coli associated with UTIs [27]. Additionally, the associations between the prevalence of IncIl and resistance to cephalosporins and between the prevalence of IncF and resistance to $\mathrm{AK}$ and SAM observed in our study indicate that plasmid IncF and IncI1 played an important role in the resistance development. Thus, the high occurrence of plasmid replicons, especially IncF and IncI1 in our study poses a serious challenge to clinical treatment based on their extensive horizontal gene transfer potential for resistance determinants between clinical strains under the selection pressure imposed by antimicrobial agents.

Novel epidemiological information pertinent to the molecular characteristics of pyelonephritis E. coli VFs is provided by our study which showed a high prevalence of fimbriae-associated adherence, indicating that adhesion of uropathogenic $E$. coli onto the epithelial cells is the first event leading to APN. Of note, the frequency of the pap gene in our study is near to the one reported by Firoozeh et al. [28] showing the important role of $\mathrm{P}$ fimbriae in the development of pyelonephritis. One of the most striking findings in our study was the high prevalence of multiple iron acquisition systems (heme/ hemoglobin gene cluster, aerobactin, and yersiniabactin), suggesting the importance of iron for the survival of UPEC in the urinary tract.

In contrast to the most commonly accepted view that resistance to quinolones is linked to a loss of VFs, [29] we found statistical associations between distribution of VFs ompT, kpsMT and levofloxacin resistant strains. The group II capsule (kpsMT) and outer membrane protease $\mathrm{T}(o m p T)$ have been frequently reported to be associated with urinary tract infections [30]. However, the correlations of these 2 VFs with resistance to floroquinolones has not been reported previously. Notably, we found that the prevalence of $\operatorname{traT}$ was associated with the production of ESBLs. This could be attributed to the occurrence of this VF and ESBL-encoding genes on the same mobile elements, which was further suggested by the association between virulence genes $\operatorname{tra} T$ and the $I n c F$ replicon. In addition, positive associations observed between the VFs and the resistance phenotypes suggest that some $E$. coli isolates associated with APN have been the reservoirs for antimicrobial resistance and virulence determinants.

Both of the PFGE and MLST in our study revealed a genetic diversity of the E. coli isolates studied, and the major STs identified has also been frequently reported to be involved in the urinary tract infections [31].

In conclusion, we found that $E$. coli isolates associated with APN showed a high frequency of resistance toward multiple antimicrobial agents routinely used during clinical treatment except imipenem and fosfomycin. Most of the isolates belonged to phylogroup D followed by B2. Phylogenetic group D strains were more resistant and less virulent than were B2 strains. A high proportion of the strains examined in this study contained plasmid replicons which may potentially facilitate the dissemination of virulence and resistance in the hospital setting. Our study brings new insight into relationships between the antimicrobial resistance, plasmid replicons, VFs, as well as phylogenetic groups in $E$. coli isolates associated with APN.

\section{Acknowledgements}

This study was supported by the Youth Fund of Jiangsu Province (Grant No. BK2014099) and the Nan Jing Medical Science and Technique Development Foundation (grant numbers: QRX11191). We are very grateful to the EU Reference Laboratory for Antimicrobial Resistance of the National Food Institute of Technical University of Denmark for providing the positive controls for our experiments.

\section{References}

1. Kim B, Kim J, Seo MR, Wie SH, Cho YK, et al. (2013) Clinical characteristics of community-acquired acute pyelonephritis caused by ESBL-producing pathogens in South Korea. Infection 41: 603-612.

2. Agarwal J, Srivastava S, Singh M (2012) Pathogenomics of uropathogenic Escherichia coli. Indian J Med Microbiol 30: 141-149.

3. Cao X, Zhang Z, Shen H, Ning M, Chen J, et al. (2014) Genotypic characteristics of multidrug-resistant Escherichia coli isolates associated with urinary tract infections. APMIS 122: 1088-1095.

4. Venturini C, Hassan KA, Chowdhury PR, Paulsen IT, Walker MJ, et al. (2013) Sequences of two related multiple antibiotic resistance virulence plasmids sharing a unique IS26-related molecular signature isolated from different Escherichia coli pathotypes from different hosts. PLoS One 8: e78862.

5. Rogers BA, Sidjabat HE, Paterson DL (2011) Escherichia coli O25bST131: a pandemic, multiresistant, community-associated strain. J Antimicrob Chemother 66: 1-14.

6. Ulett GC, Totsika M, Schaale K, Carey AJ, Sweet MJ, et al. (2013) Uropathogenic Escherichia coli virulence and innate immune responses during urinary tract infection. Curr Opin Microbiol 16: 100-107.

7. Ejrnæs K (2011) Bacterial characteristics of importance for recurrent urinary tract infections caused by Escherichia coli. Dan Med Bull 58: B4187.

8. Krieger JN, Dobrindt U, Riley DE, Oswald E (2011) Acute Escherichia coli prostatitis in previously health young men: bacterial virulence factors, antimicrobial resistance, and clinical outcomes. Urology 77: 1420-1425.

9. Clinical and Laboratory Standards Institute (2012) Performance standards for antimicrobial susceptibility testing. Twenty-Second Informational Supplement M100-S22. Wayne, PA, USA.

10. Gordon DM, Clermont O, Tolley H, Denamur E (2008) Assigning Escherichia coli strains to phylogenetic groups: multi-locus sequence typing versus the PCR triplex method. Environ Microbiol 10: 2484-2496.

11. Dallenne C, Da Costa A, Decré D, Favier C, Arlet G (2010) Development of a set of multiplex PCR assays for the detection of genes encoding important beta-lactamases in Enterobacteriaceae. J Antimicrob Chemother 65: 490-495.

12. Cattoir V, Poirel L, Rotimi V, Soussy CJ, Nordmann P (2007) Multiplex PCR for detection of plasmid-mediated quinolone resistance qnr genes in ESBL-producing enterobacterial isolates. J Antimicrob Chemother 60: 394-397.

13. Park CH, Robicsek A, Jacoby GA, Sahm D, Hooper DC (2006) Prevalence in the United States of aac(6')-Ib-cr encoding a ciprofloxacin-modifying enzyme. Antimicrob Agents Chemother 50: 3953-3955. 
Citation: Cao XL, Xu XJ, Shen H, Zhang ZF, Ning MZ, et al. (2016) Drug Resistance and Molecular Characteristics of Escherichia coli Isolates Associated with Acute Pyelonephritis. J Bacteriol Parasitol 7: 260. doi:10.4172/2155-9597.1000260

Page 6 of 6

14. Kim HB, Wang M, Park CH, Kim EC, Jacoby GA, et al. (2009) oqxAB encoding a multidrug efflux pump in human clinical isolates of Enterobacteriaceae. Antimicrob Agents Chemother 53: 3582-3584.

15. Berçot B, Poirel L, Nordmann P (2011) Updated multiplex polymerase chain reaction for detection of $16 \mathrm{~S}$ rRNA methylases: high prevalence among NDM-1 producers. Diagn Microbiol Infect Dis 71: 442-445.

16. Carattoli A, Bertini A, Villa L, Falbo V, Hopkins KL, et al. (2005) Identification of plasmids by PCR-based replicon typing. J Microbiol Methods 63: 219-228.

17. Johnson JR, Stell AL (2000) Extended virulence genotypes of Escherichia coli strains from patients with urosepsis in relation to phylogeny and host compromise. J Infect Dis 181: 261-272.

18. Ribot EM, Fair MA, Gautom R, Cameron DN, Hunter SB, et al. (2006) Standardization of pulsed-field gel electrophoresis protocols for the subtyping of Escherichia coli O157: H7, Salmonella, and Shigella for PulseNet. Foodborne Pathog Dis 3: 59-67.

19. Bouchillon SK, Badal RE, Hoban DJ, Hawser SP (2013) Antimicrobia susceptibility of inpatient urinary tract isolates of gram-negative bacilli in the United States: results from the study for monitoring antimicrobia resistance trends (SMART) program: 2009-2011. Clin Ther 35: 872-877.

20. Allocati N, Masulli M, Alexeyev MF, Di Ilio C (2013) Escherichia coli in Europe: an overview. Int J Environ Res Public Health 10: 6235-6254.

21. Lai B, Zheng B, Li Y, Zhu S, Tong Z (2014) In vitro susceptibility of Escherichia coli strains isolated from urine samples obtained in mainland China to fosfomycin trometamol and other antibiotics: a 9-year surveillance study (2004-2012). BMC Infect Dis 14: 66.

22. Mittal S, Sharma M, Chaudhary U (2014) Study of virulence factors of uropathogenic Escherichia coli and its antibiotic susceptibility pattern. Indian J Pathol Microbiol 57: 61-64.

23. Yang J, Ye L, Wang W, Luo Y, Zhang Y, et al. (2011) Diverse prevalence of $16 \mathrm{~S}$ rRNA methylase genes armA and rmtB amongst clinical multidrug- resistant Escherichia coli and Klebsiella pneumoniae isolates. Int J Antimicrob Agents 38: 348-351.

24. Mathers AJ, Peirano G, Pitout JD (2015) The role of epidemic resistance plasmids and international high-risk clones in the spread of multidrugresistant Enterobacteriaceae. Clin Microbiol Rev 28: 565-591.

25. Cao X, Cavaco LM, Lv Y, Li Y, Zheng B, et al. (2011) Molecular characterization and antimicrobial susceptibility testing of Escherichia coli isolates from patients with urinary tract infections in 20 Chinese hospitals. J Clin Microbiol 49: 2496-2501.

26. Fischer EA, Dierikx CM, van Essen-Zandbergen A, van Roermund HJ, Mevius DJ, et al. (2014) The IncI1 plasmid carrying the blaCTX-M-1 gene persists in in vitro culture of a Escherichia coli strain from broilers. BMC Microbiol 14: 77.

27. Zhang GQ, Yao YH, Yu XL, Niu JJ (2014) A survey of five broad-hostrange plasmids in gram-negative bacilli isolated from patients. Plasmid 74: 9-14.

28. Firoozeh F, Saffari M, Neamati F, Zibaei M (2014) Detection of virulence genes in Escherichia coli isolated from patients with cystitis and pyelonephritis. Int J Infect Dis 29: 219-222.

29. Basu S, Mukherjee SK, Hazra A, Mukherjee M (2013) Molecular Characterization of Uropathogenic Escherichia coli: Nalidixic Acid and Ciprofloxacin Resistance, Virulent Factors and Phylogenetic Background. J Clin Diagn Res 7: 2727-2731.

30. Kanamaru S, Kurazono H, Ishitoya S, Terai A, Habuchi T, et al. (2003) Distribution and genetic association of putative uropathogenic virulence factors iroN, iha, kpsMT, ompT and usp in Escherichia coli isolated from urinary tract infections in Japan. J Urol 170: 2490-2493.

31. Qin X, Hu F, Wu S, Ye X, Zhu D, et al. (2013) Comparison of adhesin genes and antimicrobial susceptibilities between uropathogenic and intestinal commensal Escherichia coli strains. PLoS One 8: e61169. 